\title{
SARS-CoV-2 (COVID-19) Variants and COVID-19 Vaccine Efficacy
}

\section{Cheepsattayakorn $\mathbf{A}^{1,2, *}$, Cheepsattayakorn $\mathbf{R}^{3}$ and Siriwanarangsun $\mathbf{P}^{1}$}

${ }^{1}$ Faculty of Medicine, Western University, Thailand

${ }^{2} 10^{\text {th }}$ Zonal Tuberculosis and Chest Disease Center, Thailand

${ }^{3}$ Department of Pathology, Faculty of Medicine, Chiang Mai University, Thailand

*Corresponding author: Attapon Cheepsattayakorn, 10th Zonal Tuberculosis and Chest

Research Article

Volume 6 Issue 1

Received Date: April 07, 2021

Published Date: April 22, 2021

DOI: $10.23880 /$ oajprs-16000135

Disease Center, 143 Sridornchai Road, Changklan Muang, Chiang Mai 50100, Thailand, Tel:

6653140767/6653276364; Email: Attapon1958@gmail.com

\section{Abstract}

Identification and analysis of current available COVID-19 vaccines against SARS-CoV-2 (COVID-19) variants of concern, efficacy, and their adverse events are the objective of this study. The current variants of concern, lineages B.1.351, B.1.1.7, and P1 affect the function of the spike protein and other SARS-CoV-2 proteins and can alter interaction with hACE2. The first three COVID-19 vaccines with expressing spike protein and a progressing national rollout have authorization of emergency use in the United Kingdom (UK) and demonstrated protection against COVID-19 and decreased transmission after vaccination in the preliminary report. The search was applied to the articles that were published between 2020 and early 2021. With strict literature search and screening processes, it yielded 12 articles (2020 = 2; and early 2021 to mid-March $2021=10$ articles) from 400 articles of initial literature database (2020-early 2021). A study demonstrated in early March 2021 the efficacy of various COVID-19 vaccines produced by many manufactures in symptomatic SARS-CoV-2 (COVID-19) patients and patients infected with SARS-CoV-2 (COVID-19) variants as the following products (vaccine name), used technology, doses, efficacy against symptomatic disease, and efficacy against variants (B.1.1.7 (first detected in the United Kingdom) and B.1.351 (first detected in South Africa)). It is hard to predict long term of risk of immune escape. From experience with avian coronavirus, vaccines against one variant will protect against similar variant, but not always against highly divergent variants. As SARSCoV-2 (COVID-19) variants are too divergent, similar to flu vaccines, COVID-19 vaccines will be changed. In conclusion, modifying COVID-19 vaccines would probably be the most straightforward step in involving SARS-CoV-2 (COVID-19) variants. More challenging will be deciding when and how to deploy COVID-19 vaccines 2.0.

Keywords: COVID-19; SARS-CoV-2; Variants; Vaccines; Efficacy

Abbreviations: COVID-19: Coronavirus Disease 2019; EUA: Emergency Use Authorization; hACE: human AntiConverting Enzyme; PHEIC: Public Health Emergency of International Concern; SARS-CoV-2: Severe-AcuteRespiratory-Syndrome Coronavirus type 2; UK: United Kingdom; US CDC: United States Centers for Disease Control and Prevention; WHO: World Health Organization.

\section{Introduction}

Challen, et al. indicated that B.1.1.7 variant might be related to increased mortality that supplements to the 


\section{Open Access Journal of Pulmonary \& Respiratory Sciences}

central questions of the ability of an old version of the spike glycoprotein of SARS-CoV-2 (COVID-19) to produce protective antibodies against newer emerging variants [1]. The current variants of concern, lineages B.1.351, B.1.1.7, and P1 affect the function of the spike protein and other SARS-CoV-2 proteins and can alter interaction with hACE2 [2]. The first three COVID-19 vaccines with expressing spike protein and a progressing national rollout have authorization of emergency use in the United Kingdom (UK) and demonstrated protection against COVID-19 [3-5] and decreased transmission after vaccination in the preliminary report [6].

\section{Objectives of the Study}

Identification and analysis of the efficacy and adverse events of current available COVID-19 vaccines against SARSCoV-2 (COVID-19) variants are the objective of this study.

\section{Methods of the Study}

The search was applied to the articles that were published between 2020 and early 2021. The articles were carefully selected to guarantee the literature quality, which is a tradeoff for quantity. Search string for disease groups include ["SARS-CoV-2" or "COVID-19" or "Variants" "Vaccine Efficacy" or "Vaccine Adverse Events"] in mainstream bibliographic databases or Medical Subject Headings, including PubMed, ISI Web of Science, ScienDirect, and Scopus. The initial literature databases were further manually screened with the following rules: 1)articles that did not report SARS-CoV-2 or COVID-19 related to vaccine efficacy and vaccine adverse events were not considered, such as commentary articles, or editorial; 2)non-SARS-CoV-2, non-COVID-19, non-SARSCoV-2 (COVID-19) variants-related articles were excluded; 3)duplicated and non-English articles were removed; and 4)non-peer reviewed articles were not considered to be of a scholarly trustworthy validity. The second round of publication search was conducted for adding the missing published articles that were not identified by the first round.

\section{Results}

With strict literature search and screening processes, it yielded 12 articles ( $2020=2$; and early 2021 to mid-March $2021=10$ articles) from 400 articles of initial literature database (2020-early 2021). Needed article information was extracted from each article by: 1)study period; 2)research (study) method used; 3)direct information including journal, (research article, review article, meeting abstract, conference abstract, correspondence, author index, editorial board meeting abstract, discussion), book chapter, title, authors, abstract, full text documents of candidate studies, publishing year; and 4)the conclusions made about the SARS-CoV-2
(COVID-19) vaccine efficacy and vaccine adverse events on SARS-CoV-2 (COVID-19)-variants-infected humans.

\section{Classifications and Definitions of SARS CoV-2 (COVID-19) Variants}

To remain effective against the very naturally mutated viruses by powerful adaptation of the scientific responses due to the recent emergence of variants of SARS-CoV-2 (COVID-19) [7]. The United States Center for Disease Control and Prevention (US CDC) classifies the SARS-CoV-2 (COVID-19) variants as the following [8]:

\section{Variant of Interest}

These variants are 1) B.1.526 (First detected in New York, November 2020, 2) B.1.525 (First detected in New York, December 2020, and 3) P.2 (First detected in Brazil, April 2020).

\section{Variants of Concern}

These variants are 1)B.1.1.7 (First detected in the United Kingdom, with approximately 50\% increased transmissibility, likely increased severity based on hospital admissions and case fatality rates, minimal impact on neutralization by Emergency Use Authorization (EUA) monoclonal antibody treatments), 2)P.1 (First detected in Japan or Brazil, with moderate impact on neutralization by EUA monoclonal antibody treatments), 3)B.1.351 (First detected in South Africa, with approximately $50 \%$ increased transmissibility, moderate impact on neutralization by EUA monoclonal antibody treatments), 4)B.1.427 (First detected in California, with approximately $20 \%$ increased transmissibility, significant impact on neutralization by some, but not all, EUA treatments), and 5)B.1.429 (First detected in California, with approximately $20 \%$ increased transmissibility, significant impact on neutralization by some, but not all, EUA treatments).

\section{Variant of High Consequence}

This variant has clear evidence that medical countermeasures (MCMS) or prevention measures have significant decreased effectiveness that are associated with previously circulating variants. The possible attributes that can impact on MCMS are demonstrated failure of diagnostics, evidence to indicate a significant reduction in vaccine effectiveness, a disproportionately high number of vaccine breakthrough cases, very low vaccine-induced protection against severe disease, significant decreased susceptibility to multiple EUA or approved treatments, and more severe clinical disease and increased hospital admissions. This variant would require public health officials announce a Public Health Emergency of International Concern (PHEIC). 


\section{Open Access Journal of Pulmonary \& Respiratory Sciences}

\section{Vaccine Efficacy on SARS-CoV-2 (COVID-19) Variants}

A study demonstrated in early March 2021 the efficacy of various COVID-19 vaccines produced by many manufacturers in symptomatic SARS-CoV-2 (COVID-19) patients and patients infected with SARS-CoV-2 (COVID-19) variants as the following products (vaccine name), used technology, doses, efficacy against symptomatic disease, and efficacy against variants (B.1.1.7 (first detected in the United Kingdom) and B.1.351 (first detected in South Africa)): 1)Bharat Biotech (Covaxin), inactivated virus, 2 doses, unknown, unknown, unknown; 2) Sinovac (CoronaVac), inactivated virus, 2 doses, 50.4\%, unknown, unknown; 3) Sinopharm (BBIBP-CorV), inactivated virus, 2 doses, 79.34\%, unknown, unknown (but reports of weekend effect); 4) John \& Johnson (Ad26.COV2.S), viral vector, 1 dose, 72\%, unknown, 57\%; 5) Novavax (NVX-CoV2373), protein, 2 doses, 95.6\%, 85.6\%, 60\%; 6)CanSinoBio (Convidecia), viral vector, 1 dose, 65.7\%, unknown, unknown; 7) Gamaleya (Sputnik V), viral vector, 2 doses, 91.6\%, unknown, unknown; 8) Moderna and the National Institute of Health (NIH) (mRNA1273), mRNA, 2 doses, 94.5\%, unknown (but reports of reduction in neutralizing antibodies), unknown; 9)Oxford and AstraZeneca (AZD1222), viral vector, 2 doses, 82.4\% (12 weeks between doses), 74.6\%, to be confirmed (unconfirmed reports as low as 10\%); 10)Pfizer and BioNTech (Comirnaty), mRNA, 2 doses, 95\%, unknown, and unknown; respectively [9].

\section{Discussion}

SARS-CoV-2 (COVID-19) variants of concern might be related to changes in both morbidity and mortality. Changes in both morbidity and mortality in COVID-19infected individuals may due to suppression of the host immune response, altered viral transmission dynamics, or higher viral loads that might worsen the clinical outcomes. Current COVID-19 vaccines are based on the SARS-CoV-2 spike protein, whereas the SARS-CoV-2 (COVID-19) variants contain mutations in the spike protein that contributes to spurring vaccine efficacy concerns [10]. Among 168 cases with SARS-CoV-2 (COVID-19) infection, the serious adverse events from AZD1222 was found in 79 cases in the vaccine group and 89 cases in the control group [9]. Ttransverse myelitis was identified in two cases that later determined to be unlikely to be associated [9], whereas, there is unknown serious adverse events from CoronaVac in phase III trials [9]. More serious adverse events were found in the control group than in the vaccine group [9]. Recently, The World Health Organization (WHO) recently stated that the Oxford/AstraZeneca vaccine offers protection against severe COVID-19, COVID-19-related hospitalization, and COVID-19-related death in the context without SARS-CoV-2
(COVID-19) variants, particularly B.1.351 [7]. The South Africa trials demonstrated lower vaccine efficacy compared with trials in other countries where B.1.351 variant was not dominant [10], whereas a recent study demonstrated that a two-dose regimen of the ChAdOx1 $\mathrm{nCoV}$ vaccine did not protect against mild-to-moderate B.1.351 COVID-19 variant [11]. Unfortunately, the difference of vaccine efficacy of Ad26COV2.S COVID-19 vaccine were demonstrated in the United States and South Africa (72 \% vs 57 \%) [12].

The WHO recommends on the effectiveness of COVID-19 vaccines in the context of SARS-CoV-2 (COVID-19) variants that: 1)The existing mechanism for tracking and evaluating COVID-19 variants that may affect vaccine composition must be enhanced, 2)Priority should be given to vaccinating highrisk groups everywhere, 3)Enhanced genomic surveillance must be backed by rapid haring of genetic and meta-data to allow for global coordination and response, 4)The manufacturers must be prepared to adjust to the SARS-CoV-2 (COVID-19) viral evolution, 5)Trials must be designed and maintained to allow any changes in efficacy to be assessed, and 6)Governments and donors, as well as development banks, should further support COVAX, [7].

The WHO recommendations on COVID-19 vaccines in the context of SARS-CoV-2 (COVID-19) variants contribute to the plans of the next steps on COVID-19 vaccine production, such as Novavax, whose first-generation vaccine has not been authorized yet in the United States, announced on January 28, 2021 that it was working on developing a booster, a combination bivalent vaccine, or both to protect against SARS-CoV-2 (COVID-19) variants; Moderna announced on February 24, 2021 that a booster vaccine candidate based on B.1.351 had been shipped to NIAID for a phase 1 trial; and Pfizer and BioNTech announced on February 25, 2021 that they had started evaluating the safety and immunogenicity of a third dose of their vaccine to observe whether it would boost immunity to SARS-CoV-2 (C)OVID-19) variants, particularly B.1.351; [10].

It is hard to predict long-term risk of immune escape. From experience with avian coronavirus, vaccines against one variant will protect against similar variant, but not always against highly divergent variants. As SARS-CoV-2 (COVID-19) variants are too divergent, similar to flu vaccines, COVID-19 vaccines will be changed. It might be more robust that multivalent vaccines include the viral nucleoprotein in long term.

\section{Conclusion}

Modifying COVID-19 vaccines would probably be the most straightforward step in involving SARS-CoV-2 (COVID-19) variants. More challenging will be deciding 


\section{Open Access Journal of Pulmonary \& Respiratory Sciences}

when and how to deploy COVID-19 vaccines 2.0. Much more proactively rapid identification and characterization of variants of concern will be provided by the national and global surveillance.

\section{Author's Contributions}

Dr. Attapon Cheepsattayakorn conducted the study framework and wrote the manuscript. Associate Professor Dr. Ruangrong Cheepsattayakorn and Professor Dr. Porntep Siriwanarangson contributed to scientific content and assistance in manuscript writing. All authors read and approved the final version of the manuscript.

\section{Competing Interests}

The authors declare that they have no actual or potential competing financial interests.

\section{Funding Sources}

The authors disclose no funding sources.

\section{References}

1. Challen R, Brooks-Pollock E, Read JM, Dyson L, TsanevaAtanasova K, et al. (2021) Risk of mortality in patients infected with SARS-CoV-2 variant of concern 202012/1: matched cohort study. BMJ 372: n579.

2. Yi C, Sun X, Ye J, Ding L, Liu M, et al. (2020) Key residuals of the receptor binding motif in the spike protein of SARS-CoV-2 that interact with ACE2 and neutralizing antibodies. Cell Mol Immunol 17(6): 621-630.

3. Voysey M, Clemens SAC, Madhi SA, Weckx LY, Folegatti PM, et al. (2021) Oxford COVID-19 Vaccine Trial Group.
Safety and efficacy of the ChAdOx1 $\mathrm{nCoV}-19$ vaccine (AZD1222) against SARS-CoV-2: an interim analysis of four randomized controlled trials in Brazil, South Africa, and the UK. Lancet 397(10269): 99-111.

4. Baden LR, El Sahly HM, Essink B, Kotloff K, Frey S, et al. (2021) Efficacy and safety of the mRNA-1273 SARSCoV-2 vaccine. $N$ Engl J Med; 384: 403-416.

5. Polack FP, Thomas SJ, Kitchin N, Absalon J, Gurtman A, et al. (2020) C4591001 Clinical Trial Group. Safety and efficacy of the BNT162b2 mRNA COVID-19 vaccine. N Engl J Med 383: 2603-2615.

6. Levine-Tiefenbrun M, Yelin I, Katz R, Herzel E, Golan Z, et al. (2021) Decreased SARS-CoV-2 viral load following vaccination. medRxiv.

7. World Health Organization (2021) COVAX Statement on New Variants of SARS-CoV-2.

8. United States Center for Disease Control and Prevention (2021) SARS-CoV-2 variants classifications and definitions.

9. Mahase E (2021) COVID-19: where are we on vaccines and variants? BMJ 372: n597.

10. Rubin $\mathrm{R}$ (2021) COVID-19 vaccines vs variantsdetermining how much immunity is enough. JAMA 325(13): 1241-1243.

11. Madhi SA, Baillie CL, Cutland M, Voysey AL, Koen L, et al. (2021) Efficacy of the ChAdOx1 nCoV-19 COVID-19 Vaccine against the B.1.351 Variants. N Engl J Med 14.

12. Cohen J (2021) One-dose of COVID-19 vaccine offers solid protection against severe disease. Science. 